\title{
Optical Observation of Vortex-Flame Interaction Using Spark-Ignited Premixed Flames in a Quasi-Turbulent and Quiescent Wake*
}

\author{
Yojiro ISHINO**, Yasunori HATTORI*** \\ and Norio OHIWA**
}

\begin{abstract}
Structure and behavior of spark-ignited propane-air premixed flames established in the quasi-turbulent and quiescent wake, which is generated by quickly moving a fine rod-array vertically downward in the quiescent stoichiometric propane-air mixture, are observed and analyzed using tomography and PTV. It is found that the quasi-turbulent and quiescent wake proposed here is composed of Karman vortex streets with the characteristic length scale ranging $0.2 \sim 0.5 \mathrm{~mm}$ and the tangential velocity of $4.0 \mathrm{~m} / \mathrm{s}$, and therefore that it satisfies rather small and intense turbulence conditions of $l_{\mathrm{d}} / \delta_{\mathrm{L}} \approx 1$ and $v_{\theta} / S_{\mathrm{L}} \approx 10$. Since the interface between the unburnt and burnt gases exhibits complicated and rugged appearances and consists of a continuous series of wrinkled flame elements of the length scale ranging $0.2 \sim 2 \mathrm{~mm}$, the turbulent flame established in the quasi-turbulent and quiescent wake with the moderately intense turbulence conditions of $l_{\mathrm{d}} / \delta_{\mathrm{L}} \approx 1$ and $v_{\mathrm{\theta}} / S_{\mathrm{L}} \approx 10$ is classified into the finely wrinkled laminar flame.
\end{abstract}

Key Words: $\quad$ Turbulent Premixed Flame, Wrinkled Laminar Flame, Tomography, PTV, CH-Emission, Wake, Karman Vortex, Spark Ignition

\section{Introduction}

Structure of turbulent premixed flames is wellknown to be greatly affected by the turbulence characteristics of the mixture flow. Some typical models of the turbulent flame structure ${ }^{(1),(2)}$ are proposed using the nondimensional characteristic parameters; $u^{\prime} / S_{\mathrm{L}}$ and $l / \delta_{\mathrm{L}}$, where $u^{\prime}$ and $S_{\mathrm{L}}$ indicate the turbulence intensity and the laminar burning velocity, and $l$ and $\delta_{\mathrm{L}}$ the turbulence length scale and the laminar flame thickness, respectively. Depending on whether $u^{\prime} / S_{\mathrm{L}}<<1$ and $l / \delta_{\mathrm{L}}>1$ or $u^{\prime} / S_{\mathrm{L}}>1$ and $\| / \delta_{\mathrm{L}}<1$, two extreme flame models are imaged; the wrinkled laminar flame model and the distributed reaction zone model, respectively. With respect to the

* Received April 30, 2000

** Department of Mechanical Engineering, Nagoya Institute of Technology, Gokiso-cho, Showa-ku, Nagoya 466-8555, Japan

*** Tamano Works, Mitsui Engineering \& Shipbuilding Co., Ltd. flame structure in the intermediate condition between two extremes, however, no consistent and reasonable model is proposed, although much attention is focused from the theoretical and practical points of view. This is because that the intermediate condition includes practically too wide range of turbulence properties to make detailed observation of the vortex-flame interaction under consistently subdivided ranges of turbulence conditions. In order to constitute a consistent set of turbulent flame models in the intermediate range of turbulence conditions between two extremes, therefore, further experimental observations and analyses should be systematically made of the interdependency between the concrete processes of the vortex-flame interaction and the subdivided turbulence conditions, for example, by varying the characteristics length scale as $l / \delta_{\mathrm{L}}>1, l / \delta_{\mathrm{L}} \approx 1$, and $l / \delta_{\mathrm{L}}<1$ under the moderately intense turbulence condition of $u^{\prime} / S_{\mathrm{L}}>1$.

From the point of view mentioned above, the main objective of this investigation is to examine experimentally the interactions between a laminar 
combustion wave with a cluster of vortices, not with a single vortex, under the subdivided turbulence conditions of $u^{\prime} / S_{\mathrm{L}}>1$ and $l / \delta_{\mathrm{L}} \approx 1$. In order to simplify observation and explanation of the flame structure and behavior, a wake behind a quickly downward moved rod-array in the quiescent mixture, which is apparently two-dimensional and is composed of an alternatively arranged regular array of Karman vortex streets having nearly equal length scale with each other, is first selected as a turbulent flow field where flames are established. This flow is named "the quasi-turbulent and quiescent wake" in this investigation, for lack of randomness and three dimensionality particular to turbulence.

A stoichiometric propane-air premixed flame is initiated in the quasi-turbulent and quiescent wake by a spark ignition and interacts with an array of vortex tubes, as it propagates radially in the wake. In this paper concrete time histories of the vortex-flame interaction are optically observed and analyzed. Profiles of $\mathrm{CH}$-emission intensity fluctuation of the propagating flames are also measured. In the optical observations an original device is introduced for easy and clear explanations of the vortex-flame interaction.

\section{Nomenclature}

$d_{0}$ diameter of rod element of the rod-array $(\mathrm{mm})$

$d_{\mathrm{p}}$ diameter of incense smoke particles $\quad(\mathrm{mm})$

$f_{0}$ formation frequency of Karman vortex street

$f_{\theta} \quad$ tangential frequency of vortex tube

$l$ turbulence length scale $l_{\mathrm{d}} \quad$ mean diameter of quasi-turbulence vortex $(\mathrm{mm})$

$S_{\mathrm{F}} \quad$ flame speed $(\mathrm{m} / \mathrm{s})$

$S_{\mathrm{L}} \quad$ measured laminar burning velocity $\quad(\mathrm{m} / \mathrm{s})$

$S_{\mathrm{Lm}}$ average laminar burning velocity $\quad(\mathrm{m} / \mathrm{s})$

$S_{\mathrm{t}} \quad$ Strouhal number

$t$ time after spark ignition

$t_{1 / 2}$ half life time of vortex tangential velocity $(\mu \mathrm{s})$

$U_{\mathrm{g}}$ average rod-array velocity $\quad(\mathrm{m} / \mathrm{s})$

$u^{\prime}$ turbulence intensity $(\mathrm{m} / \mathrm{s})$

$V_{\mathrm{p}}$ output of photomultiplier

$v_{\theta} \quad$ tangential velocity of vortex tube

$\delta_{\mathrm{L}}$ thickness of laminar premixed flame $\quad(\mathrm{mm})$

$v$ kinematic viscosity $\left(\mathrm{m}^{2} / \mathrm{s}\right)$

$\rho_{\mathrm{b}}$ densities of burnt gas $\quad\left(\mathrm{kg} / \mathrm{m}^{3}\right)$

$\rho_{\mathrm{p}}$ mean density of incense smoke particles $\left(\mathrm{kg} / \mathrm{m}^{3}\right)$

$\rho_{\mathrm{u}}$ densities of unburnt gas $\quad\left(\mathrm{kg} / \mathrm{m}^{3}\right)$

\section{Experimental Apparatus and Methods}

The schematic diagram of the experimental apparatus is presented in Fig. 1, and structural details and dimensions of a rod-array and a set of spark needles are also shown in Fig. 2. The apparatus consists of a combustion test section including the rod-array, a rod-array driving unit, a mixture supply line, an optical system for $\mathrm{CH}$-emission intensity measurement, and two types of optical observation systems. As shown in Fig.2, the rod-array is constructed using six parallel cylindrical rods of 0.3 $\mathrm{mm}$ diameter, $8.0 \mathrm{~mm}$ length, and $0.6 \mathrm{~mm}$ spacing, and is fixed to the exit section of a rectangular pipe, which has $5.0 \mathrm{~mm} \times 5.0 \mathrm{~mm}$ inner sectional area, 6.0 $\mathrm{mm} \times 6.0 \mathrm{~mm}$ outer sectional area, and $550 \mathrm{~mm}$ length

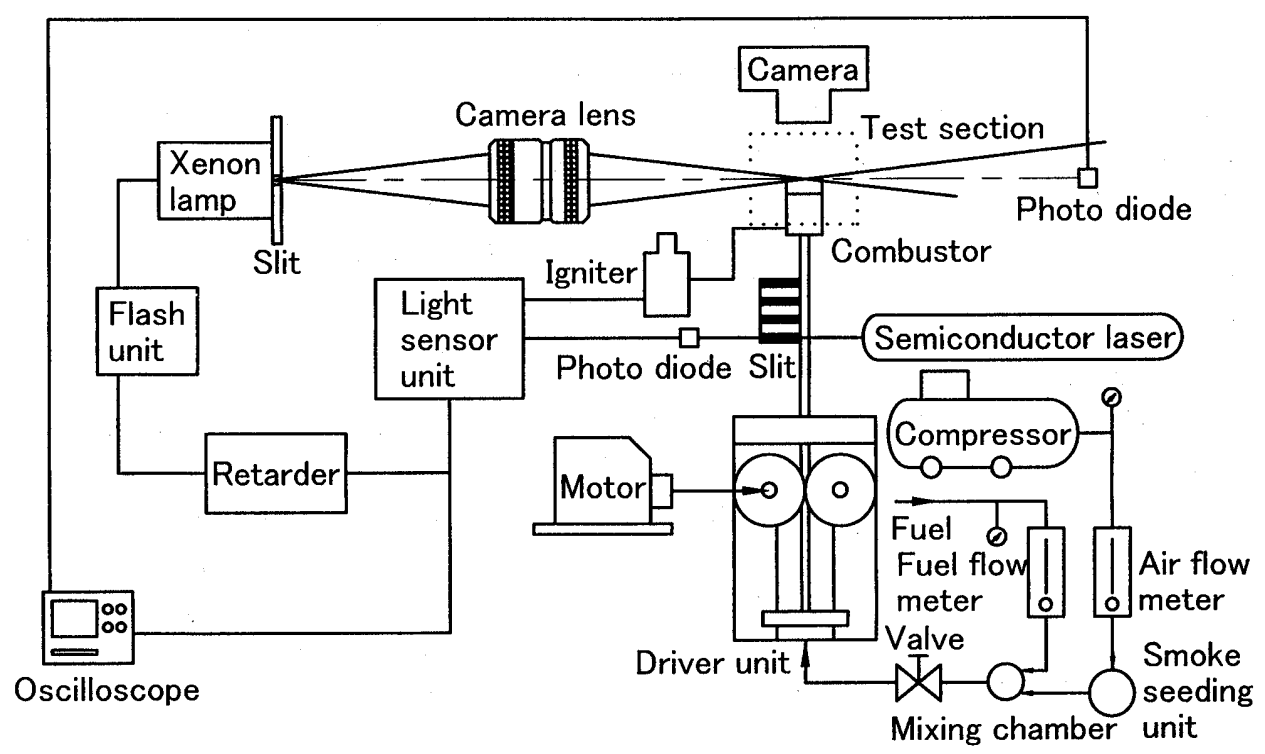

Fig. 1 Schematic diagram of the experimental apparatus 
A set of spark needles, made of brass wire of $0.3 \mathrm{~mm}$ diameter and $2.6 \mathrm{~mm}$ length and have a gap of $5.0 \mathrm{~mm}$, are installed at the central position $4.0 \mathrm{~mm}$ upward from the exit section with the axis parallel to rods.

Air supplied by a blower and propane from a bomb (commercial grade gaseous propane of $96.4 \%$ purity) are metered individually and mixed to form the stoichiometric mixture, and filled into the rectangular pipe. After being filled with the stoichiometric propane-air mixture, the rectangular pipe with the rod-array at the top end is rapidly moved vertically downward by the driving unit. Triggered by a signal generated from the laser light system, which is composed of a semiconductor laser, a light shield plate with three transparent strips of $5.75 \mathrm{~mm}$ spacings, a photo-diode, and a light sensor unit, the spark needles are discharged in the quasi-turbulent and quiescent wake, resulting in a laminar or turbulent combustion wave. Three typical stages of one combustion experiment are illustrated in Fig.3; (a) filling-up of mixture, (b) rapid downward movement of the rod-array and formation of the quasi-turbulent and quiescent wake, and (c) flame propagation after ignition. $U_{\mathrm{g}}$ in the figure indicates the average rod-array velocity of downward movement and is calculated by dividing the spacing of strips of 5.75 $\mathrm{mm}$ by an average time interval detected by the laser

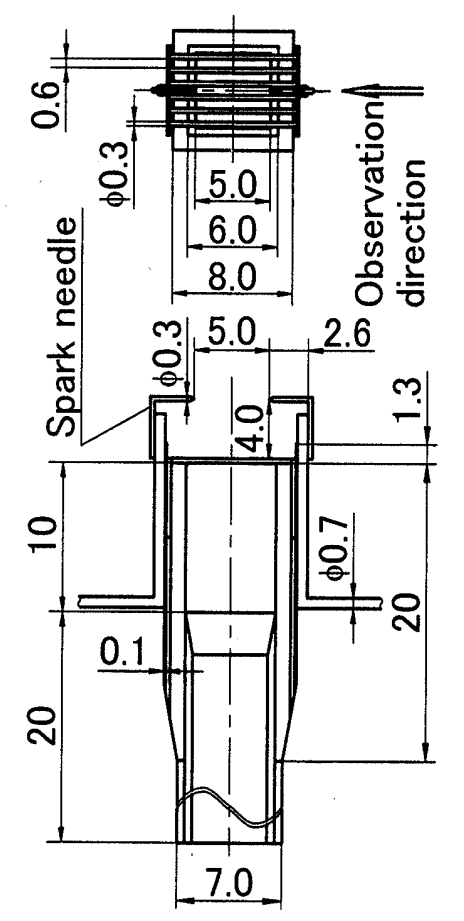

Fig. 2 Construction and dimensions of a rod-array and an ignition system employed in this experiment light system. The ignition energy discharged is 25.4 $\mathrm{mJ}$, being much greater than the minimum ignition energy of $0.4 \mathrm{~mJ}$ for the stoichiometric propane-air mixture $^{(3)}$.

Time histories of $\mathrm{CH}$-emission intensity from the propagating flame after spark ignition are measured with a detection unit consisting of a photomultiplier tube (R928; a range of sensitive wave length of 185 $900 \mathrm{~nm}$ and a maximum sensitivity at $400 \mathrm{~nm}$ ) and two kinds of optical filters; one is an interference filter having a central wave length of $426.8 \mathrm{~nm}$, a halfwidth of $14 \mathrm{~nm}$, and a maximum transmissivity of $33.7 \%$, and the other is a usual blue glass filter. The latter filter is employed to eliminate the influences of background light noise on the quantitative estimation concerning whether $\mathrm{CH}$-light emission is detected.

Optical observations of the wake structure and the behavior of combustion waves are carried out with two types of optical methods; tomography and PTV (Particle Track Velocimetry). In the tomography analysis a xenon flash light beam with $22 \mu$ s pulse width passes through a narrow slit and a lens system, and is focused into a light sheet of $1 \mathrm{~mm}$ thickness and $25 \mathrm{~mm}$ height on the central section perpendicular to the rod axis. In PTV two consecutive sets of xenon flash lamps having different pulse widths of $38 \mu$ s and $13 \mu \mathrm{s}$ and an interval of $50 \mu \mathrm{s}$ are combined with the same lens system that used in the tomography method, so that easy and reliable determination of the particle direction and speed is realized. In all cases the first xenon flash can be triggered at an arbitrary delay time after ignition by the same signal that used to trigger the spark discharge.

Smoke particles of an incense stick are used as tracer particles in the tomography and PTV

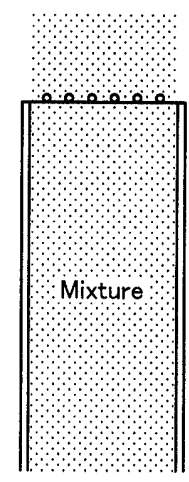

(a)

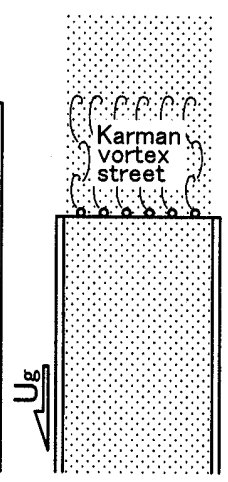

(b)

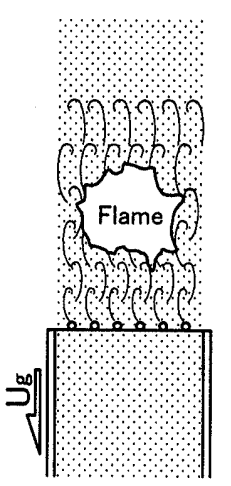

(c)
Fig. 3 Illustrations of the formation process of a quasi-turbulent and quiescent wake and a turbulent premixed propagating flame after spark ignition 
Table 1 Specifications of incense smoke particles

\begin{tabular}{c|c|c}
\hline $\begin{array}{c}\text { Particle Diameter } \\
d \mathrm{p}[\mu \mathrm{m}]\end{array}$ & $\begin{array}{c}\text { Average Density } \\
\rho_{\mathrm{p}}\left[\mathrm{kg} / \mathrm{m}^{3}\right]\end{array}$ & $\begin{array}{c}\text { Responsibility } \\
{[\%]}\end{array}$ \\
\hline $0.1 \sim 1.0$ & $2.2 \times 10^{3}$ & 93.2 \\
\hline
\end{tabular}

observations. In Table 1 specifications of the smoke particles are shown, together with the amplitude response characteristics to the virtual flow oscillation at $7.4 \mathrm{kHz}$, which corresponds to Karman vortex shedding frequency behind a rod of $0.3 \mathrm{~mm}$ diameter in a uniform velocity stream of $U_{\mathrm{g}}=10 \mathrm{~m} / \mathrm{s}$ and will be evaluated later in this paper. It is found that the smoke particles have diameters ranging $d_{\mathrm{p}}=0.1 \sim 1.0$ $\mu \mathrm{m}$ and an average density of $\rho_{\mathrm{p}}=2.2 \times 10^{3} \mathrm{~kg} / \mathrm{m}^{3}$, and can follow to the imposed flow oscillation at a remarkably high probability more than $90 \%$.

\section{Results and Discussions}

\subsection{Approximate evaluation of half-life time of quasi-turbulence vortices}

In order to obtain clear images of the vortexflame interaction, it is first necessary to initiate and propagate a combustion wave in the wake region where sound vortices with moderate tangential velocity prevail. Approximate evaluation of the half-life time of the Karman vortex tube is then attempted so that the time duration suitable for clear observation of the vortex-flame interaction can be quantitatively determined.

Since such small vortices as those generated in this investigation are greatly affected by the viscosity, Oseen's treatment of a single vortex tube ${ }^{(4)}$ can be adopted. By denoting the initial vortex diameter by $d_{0}$ and the kinematic viscosity by $v$, the half-life time of the vortex tangential velocity is finally given by $t_{1 / 2}=$ $3 d_{0}^{2} / 16 v$. Assuming the initial vortex diameter to be equal to the rod diameter; $d_{0}=0.3 \mathrm{~mm}$, using the kinematic viscosity of air at STP; $v=1.56 \times 10^{-5} \mathrm{~m}^{2} / \mathrm{s}$, and substituting these values into the above equation, the half-life time is calculated to be $t_{1 / 2}=1.08 \mathrm{~ms}$. It is found that observation of the vortex-flame interaction should be carried out within a short time duration less than $1 \mathrm{~ms}$. According to this simplified analysis, optical observations in this investigation are limited to the initial short time duration of about $600 \mu \mathrm{s}$ after ignition.

\subsection{Properties of the quasi-turbulent and quiescent wake}

In Fig.4 (a) and (b), a set of a PTV-image and an instantaneous vector diagram of the cold quasiturbulent and quiescent wake without flame are shown, respectively, where the average rod-array velocity is $U_{\mathrm{g}}=10.6 \mathrm{~m} / \mathrm{s}$. Particle tracks in Fig.4 (a) indicate that alternately arranged and relatively regular Karman vortex streets prevail in the wake behind the vertically downward moved rod-array. In the region $4 \sim 10 \mathrm{~mm}$ downstream of the rod-array, furthermore, where premixed flames are established, small scale vortices originated from Karman vortex streets exist over the wake zone. According to picture processing of the vector diagram presented in Fig.4 (b), quantitative details of the quasi-turbulence properties can be

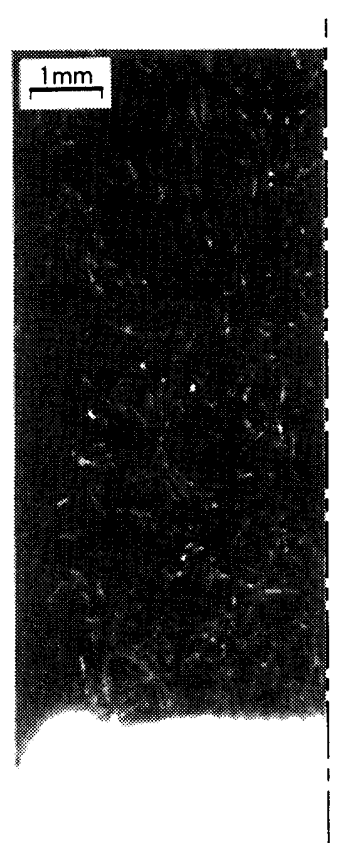

(a) PTV-image

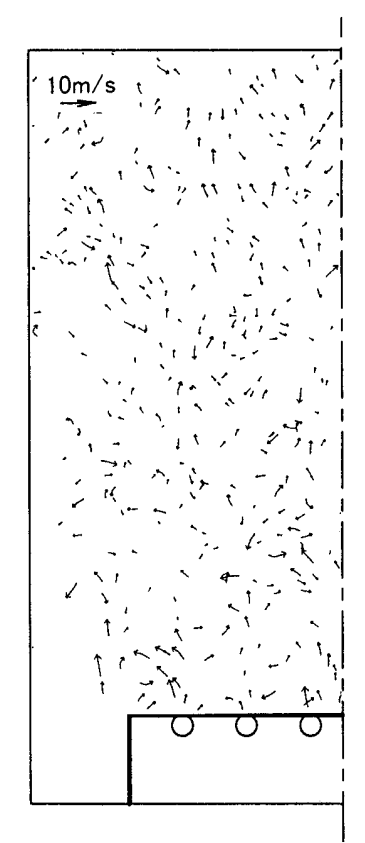

(b) Vector diagram
Fig. 4 A set of (a) a PTV-image and (b) a vector diagram of the cold quasi-turbulent and quiescent wake, where the rod-array velocity is $U_{\mathrm{g}}=10.6 \mathrm{~m} / \mathrm{s}$

Table 2 Properties of the quasi-turbulence vortex tubes

\begin{tabular}{c|c|c|c|c}
\hline $\begin{array}{c}\text { Vortex Diameter } \\
l_{\mathrm{d}}[\mathrm{mm}]\end{array}$ & $\begin{array}{c}\text { Tangential Velocity } \\
\nu_{\theta}[\mathrm{m} / \mathrm{s}]\end{array}$ & $\begin{array}{c}\text { Tangential Frequency } \\
f_{\theta}[\mathrm{kHz}]\end{array}$ & $\begin{array}{c}\text { Scale Ratio } \\
l_{\mathrm{d}} / \delta_{\mathrm{L}}\end{array}$ & $\begin{array}{c}\text { Velocity Ratio } \\
v_{\theta} / S_{\mathrm{L}}\end{array}$ \\
\hline \hline 0.3 & 4.0 & 4.2 & 1 & 10 \\
\hline
\end{tabular}


estimated and are summarized in the left three columns in Table 2 . The characteristic diameter, the tangential velocity and the tangential frequency are shown to take values of $l_{\mathrm{d}} \approx 0.3 \mathrm{~mm}, v_{\theta}=4.0 \mathrm{~m} / \mathrm{s}$, and $f_{\theta}\left(=v_{\theta} / \pi l_{\mathrm{d}}\right)=4.2 \mathrm{kHz}$, respectively.

By assuming that Strouhal number takes a value of $S_{\mathrm{t}}=0.21^{(5)}$ for the wake behind a single rod of 0.3 $\mathrm{mm}$ diameter in the uniform stream of a velocity of $U_{\mathrm{g}}=10.6 \mathrm{~m} / \mathrm{s}$, the shedding frequency of Karman vortex is calculated to be $f_{0}=7.4 \mathrm{kHz}$. Also assuming that the tangential velocity of Karman vortex approximately corresponds to the maximum turbulence intensity in the wake, which takes a value of $20 \sim$ $40 \%$ of the uniform velocity ${ }^{(6)}$, it can be evaluated to be $v_{\theta}=2.1 \sim 4.2 \mathrm{~m} / \mathrm{s}$. These values exhibit reasonable agreement with those obtained above.

\subsection{Time variations of $\mathrm{CH}$-emission intensity from propagating flames}

In order to discuss the appearances and behavior of the combustion waves based on optical observations, it is indispensable to certify first whether the interfaces focused on the tomography images really correspond to the flame surfaces. Since the detection of $\mathrm{CH}$-light emission in the hydrocarbon-fueled combustion is equivalent to the existence of the reacting flame, the intensity fluctuation of $\mathrm{CH}$-emission from the propagating flame after the spark ignition is measured. The results are shown in Fig. 5 in the form of time history, where a solid line indicates $\mathrm{CH}$-emission intensity profile of a

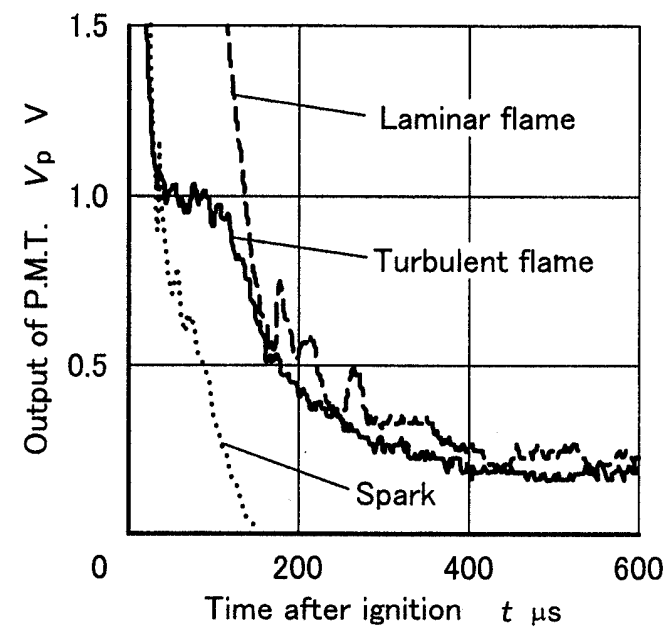

Fig. 5 Time histories of $\mathrm{CH}$-emission intensity, where a solid line indicates that of the spark- ignited flame in the quasi-turbulent and quiescent wake with $U_{\mathrm{g}}=10.5 \mathrm{~m} / \mathrm{s}$, a dashed line gives that of the spark-ignited laminar flame in the quiescent mixture, and a dotted line presents that of a spark in an atmosphere spark-ignited turbulent flame in the quasi-turbulent and quiescent wake with $U_{\mathrm{g}}=10.5 \mathrm{~m} / \mathrm{s}$, a dashed line gives that of a spark-ignited laminar flame propagating in the quiescent mixture, and a dotted line presents that of an electric spark in the atmosphere without combustion.

Since no signal, such as electric noise, is detected when the perfect optical shield is made on the photomultiplier, it is found that an electric spark in the atmosphere generates definite but quickly damping light around a wave length of $\mathrm{CH}$-emission in a short period of about $100 \mu \mathrm{s}$. Compared with the spark light emission given by the dotted line, the profiles of $\mathrm{CH}$-emission from laminar and turbulent flames exhibit enough intensity to certify the existence of the flame during the observation period less than $600 \mu \mathrm{s}$ after the spark ignition. In the following, therefore, examinations and discussions of the experimental results are made by considering the interfaces observed in the tomography images as the propagating flame surfaces.

Further comparison of the profile of $\mathrm{CH}$-emission intensity of a laminar flame with that of a turbulent one reveals that the former emits more intense $\mathrm{CH}$-light in the earlier stage of the flame propagation than the latter. This suggests strong cooling effect by the quasi-turbulence vortices on the incipient formation stage of a sound combustion wave.

\subsection{Tomography observations of spark-ignited laminar and turbulent flames}

Five sets of instantaneous tomograph images of a laminar combustion wave formed in the quiescent mixture and those of a turbulent combustion wave propagating in the quasi-turbulent and quiescent wake, which are taken from the direction parallel to the rod axis at time intervals of about $100 \mu \mathrm{s}$, are shown in Fig.6 (a) and (b), respectively, where the average rodarray velocity in Fig. 6 (b) is $U_{\mathrm{g}}=10.5 \mathrm{~m} / \mathrm{s}$. As schematically described in Fig.6, the zone of white image indicates the unburnt mixture region, the leftand the right-side black zones give the atmospheric region, and the central black zone with a smooth or a rugged interface at its surroundings gives the burnt gas region.

A spherically expanding combustion wave with a smooth interface can be observed in each image in Fig.6 (a), being particular to the laminar premixed flame. The propagation velocity of the combustion wave, which is called the flame speed and denoted by $S_{\mathrm{F}}$, is then estimated to be $S_{\mathrm{F}}=2.1 \sim 4.5 \mathrm{~m} / \mathrm{s}$. Assuming here the density ratio to be $\rho_{\mathrm{u}} / \rho_{\mathrm{b}}=6.0$, where $\rho_{\mathrm{u}}$ and $\rho_{\mathrm{b}}$ indicate densities of the unburnt mixture and burnt gas, respectively, yields the laminar burning velocity 


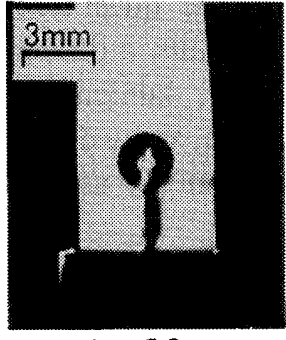

$t=98 \mu \mathrm{s}$

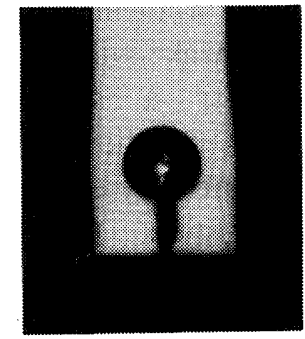

$t=208 \mu \mathrm{s}$

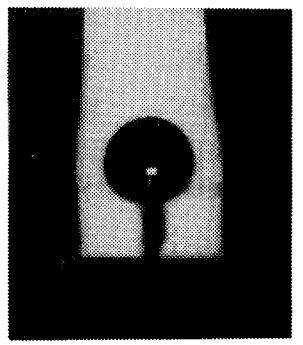

$t=308 \mu \mathrm{s}$

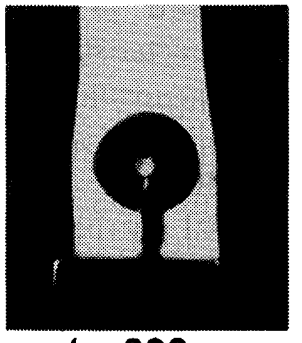

$t=396 \mu \mathrm{s}$

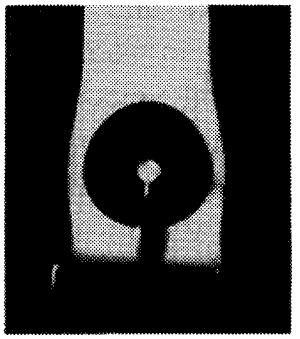

$t=506 \mu s$

(a) Tomograph images of a laminar flame propagation in the quiescent mixture

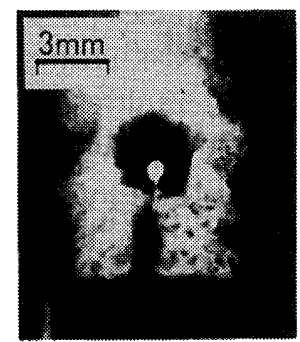

$t=100 \mu \mathrm{s}$

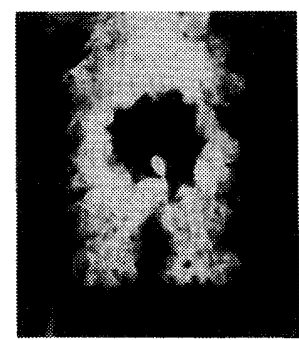

$t=208 \mu \mathrm{s}$

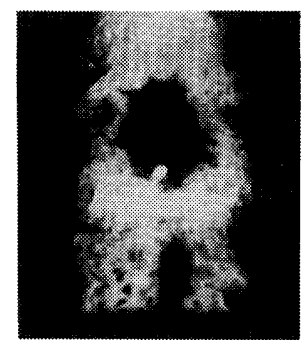

$t=300 \mu \mathrm{s}$

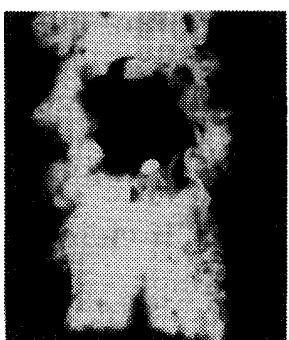

$t=406 \mu \mathrm{s}$

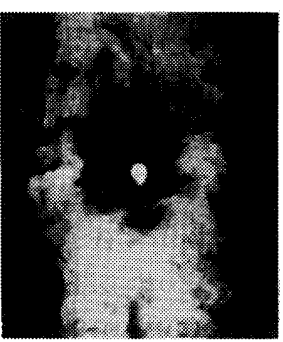

$t=496 \mu \mathrm{s}$

(b) Tomograph images of a turbulent flame propagation in the quasi-turbulent and quiescent wake

Fig. 6 Five sets of tomograph images of (a) propagating flames in the quiescent mixture, and (b) propagating flames in the quasi-turbulent and quiescent wake, where the average rod-array velocity is $U_{\mathrm{g}}=10.5 \mathrm{~m} / \mathrm{s}$

ranging $S_{\mathrm{L}}=35 \sim 75 \mathrm{~cm} / \mathrm{s}$ for the stoichiometric propane-air mixture, indicating reasonable agreement with that obtained experimentally by Harries, M. E. et $\mathrm{al}^{(7)}$.

Images of the turbulent flame formed in the quasi-turbulent and quiescent wake given in Fig.6 (b), on the other hand, show that a smooth circular laminar combustion wave is completely crumpled up by the quasi-turbulence vortices into a complex and wrinkled interface between the unburnt and burnt gases. It is also found that the rugged interface between the white zone and the central black zone is composed of a series of wrinkles with the length scale of $0.2 \sim 2 \mathrm{~mm}$, being of same order as that of the quasi-turbulence vortices. Therefore, further analysis of the concrete relationship between the flame properties and the quasi-turbulence characteristics is expected to provide some key factors for elucidating the enhancement mechanism of the flame propagation by the vortex-flame interaction. Taking into account of the experimental certification by $\mathrm{CH}$-emission measurements, the wrinkled interface is found to correspond to the propagating flame surface and is classified into the finely wrinkled laminar flame.

3.5 Relation between turbulence characteristics and flame structure

Detailed observation of Fig. 6 (b) exhibits an important characteristic feature with respect to the quasi-turbulent and quiescent wake employed in this investigation. Karman vortex streets can be clearly distinguished in the lower part of the white zone as an alternative array of small black points, and their diameters are measured to have almost same values ranging $l_{\mathrm{d}}=0.2 \sim 0.5 \mathrm{~mm}$ as that of the rod diameter of $0.3 \mathrm{~mm}$. This results in a rather small length scale of $l_{\mathrm{d}} / \delta_{\mathrm{L}} \approx 1$. On the other hand, the quantitative estimation of the vortex tangential velocity, given by $v_{\theta}=4.0 \mathrm{~m} / \mathrm{s}$, and the laminar burning velocity, ranging $S_{\mathrm{L}}=35 \sim 75 \mathrm{~cm} / \mathrm{s}$ and being averaged to be $S_{\mathrm{Lm}}=57 \mathrm{~cm} / \mathrm{s}$, results in a relatively intense turbulence condition of $v_{\theta} / S_{\mathrm{Lm}}=7 \sim 10$. These values are listed in the right two columns of Table 2 .

It is summarized here that the proposed apparatus can realize such a valuable turbulence field that consists of an array of small and moderately intense vortex tubes. They have a rather small characteristic length scale nearly equal to the laminar flame thickness and a relatively intense vortex strength about an order of magnitude greater than the laminar burning velocity; $l_{\mathrm{d}} / \delta_{\mathrm{L}} \approx 1$ and $v_{\theta} / S_{\mathrm{L}} \approx 10$. It is concluded therefore that, by interacting with an array of vortex tubes having rather small and intense turbulence conditions of $l_{\mathrm{d}} / \delta_{\mathrm{L}} \approx 1$ and $\nu_{\theta} / S_{\mathrm{L}} \approx 10$, a smooth laminar combustion wave is crumpled up into 
a finely wrinkled laminar flame consisting of a continuous series of complex and rugged flame elements.

\section{Concluding Remarks}

In this paper, spark-ignited turbulent premixed flames are established in the quasi-turbulent and quiescent wake, which is formed by rapidly moving the rod-array vertically downward in the stoichiometric propane-air mixture. Tomography and PTV are used to observe the structure and behavior of propagating flames. Measurements of $\mathrm{CH}$-emission intensity are also carried out. Observations and analyses are restricted to the initial short time duration of $600 \mu \mathrm{s}$ after the spark ignition, according to the approximate quantitative evaluation of the half-life time of the quasi-turbulence vortex. The results are summarized as follows.

(1) The quasi-turbulent and quiescent wake is found to be composed of an alternative array of Karman vortex streets having almost same length scale ranging $l_{\mathrm{d}}=0.2 \sim 0.5 \mathrm{~mm}$ as that of the rod diameter of $d_{0}=0.3 \mathrm{~mm}$. This results in a rather small turbulence length scale of $l_{\mathrm{d}} / \delta_{\mathrm{L}} \approx 1$.

(2) Based on PTV measurements, the tangential velocity of the quasi-turbulence vortex tubes is estimated to be $v_{\theta}=4.0 \mathrm{~m} / \mathrm{s}$. The mean laminar burning velocity is also evaluated to be $S_{\mathrm{Lm}}=57 \mathrm{~cm} / \mathrm{s}$ by processing the tomography images. This results in a rather intense turbulence condition of $v_{\theta} / S_{\mathrm{L}} \approx 10$. The proposed quasi-turbulent and quiescent wake is therefore found to produce the moderately intense turbulence conditions of $l_{\mathrm{d}} / \delta_{\mathrm{L}} \approx 1$ and $v_{\theta} / S_{\mathrm{L}} \approx 10$.

(3) Interacting with an array of quasi-turbulence vortex tubes as it propagates in the quasi-turbulent and quiescent wake, a smooth laminar combustion wave is crumpled up into a complex and rugged interface consisting of wrinkles of the length scale ranging $0.2 \sim 2 \mathrm{~mm}$. It is concluded that the turbulent flame established in the quasi-turbulent and quiescent wake with the moderately intense turbulence conditions; $l_{\mathrm{d}} / \delta_{\mathrm{L}} \approx 1$ and $v_{\theta} / S_{\mathrm{L}} \approx 10$, is classified into the finely wrinkled laminar flame.

\section{Acknowledgments}

The authors wish to thank Mr. Atsushi TACHI and Mr. Takahiro YAMASHINA for their faithful contribution during this investigation.

\section{References}

(1) Ballal, D. R. and Lefebvre, A. H., Proceedings of Royal Society of London, Ser. A, Vol.344, (1975), p.217-234.

(2) Poinsot, T., Veynante, D., and Candel, S., Journal of Fluid Mechanics, Vol.288, (1991), p.561-606.

(3) Lewis, B. and von. Elbe, G., Combustion, Flames and Explosions of Gases, 3rd ed., Academic Press, (1987), p.345.

(4) Aihara, Y., Mechanics of Fluid Flow (in Japanese), Baifukan, 1st ed., (1984), p.53.

(5) Schlichting, H., (Translated by Kestin, J.), Boundary-Layer Theory, 7th ed., McGraw-Hill, (1979), p.31.

(6) Kumada, M., Hiwada, M., Ito, M. and Mabuchi, I., Transactions of the JSME (in Japanese), Vol.50, No.455B, (1984), p.1699-1706.

(7) Harris, M. E., Grumer, J., von. Elbe, G. and Lewis, B., Proceedings of 3rd Symposium (International) on Combustion, Flame and Explosion Phenomena, Williams \& Wilkins, Baltimore, (1949), p.80-89. 